\title{
仿生金属配合物催化的 $\mathrm{C}\left(\mathrm{sp}^{3}\right)-\mathrm{H}$ 键不对称氧化反应研究进展
}

\author{
孙强盛孙伟* \\ (中国科学院兰州化学物理研究所 羰基合成与选择氧化国家重点实验室 \\ 分子合成科学卓越创新中心 兰州 730000)
}

\begin{abstract}
摘要 $\mathrm{C}-\mathrm{H}$ 氧化反应是一类重要的有机反应, 其中 $\mathrm{C}-\mathrm{H}$ 不对称氧化反应可以将简单烃类化合物直接转化为手性醇和 酮等, 为复杂分子的合成提供了更加经济和高效的方法. 尽管在过去的数十年, 很多化学家致力于不对称 $\mathrm{C}-\mathrm{H}$ 氧化反 应研究, 但由于 $\mathrm{C}-\mathrm{H}$ 键本身的惰性和 $\mathrm{C}-\mathrm{H}$ 键之间微弱的立体性差异, 该研究领域的进展依然缓慢. 结合影响 $\mathrm{C}-\mathrm{H}$ 氧化反应的因素、生物酶催化氧化机制与仿生金属配合物催化剂的发展，从配体类型的角度阐述当前所实现的 $\mathrm{C}-\mathrm{H}$ 键不对称氧化的一些反应实例.
\end{abstract}

关键词 $\mathrm{C}-\mathrm{H}$ 氧化; 金属配合物; 不对称氧化

\section{Recent Progress in $\mathrm{C}\left(\mathrm{sp}^{3}\right)-\mathrm{H}$ Asymmetric Oxidation Catalyzed by Bioinspired Metal Complexes}

\author{
Sun, Qiangsheng Sun, Wei* \\ (State Key Laboratory for Oxo Synthesis and Selective Oxidation, Center for Excellence in Molecular Synthesis, \\ Lanzhou Institute of Chemical Physics, Chinese Academy of Sciences, Lanzhou 730000)
}

\begin{abstract}
C}-\mathrm{H}$ oxidation represents one of the most important reactions in organic chemistry. In particular, asymmetric C$\mathrm{H}$ oxidation, which can directly convert simple alkanes into chiral alcohols, ketones, aldehydes and so on, provides more economic and efficient access to the synthesis of complex molecules. Although increasing efforts have been devoted to this area, asymmetric $\mathrm{C}-\mathrm{H}$ oxidation is still far away from the goal due to the inert nature of $\mathrm{C}-\mathrm{H}$ and the subtle stereo-difference of $\mathrm{C}-\mathrm{H}$ bonds. The factors that dictate the selectivity of asymmetric $\mathrm{C}-\mathrm{H}$ oxidation, mechanism of the $\mathrm{C}-$ $\mathrm{H}$ oxidation catalyzed by enzyme and some successful examples achieved by biomimetic metal complexes bearing various ligands are reviewed.
\end{abstract}

Keywords C $-\mathrm{H}$ oxidation; metal complex; asymmetric oxidation

$\mathrm{C}-\mathrm{H}$ 键直接氧化反应可在烷烃底物中引入氧原子, 将其直接变为醇、酮或醛等重要含氧化合物，是非常重 要的有机反应, 用途广泛 ${ }^{[1]}$. 其中, 烷烃 $\mathrm{C}-\mathrm{H}$ 键的不对 称氧化反应是极具挑战性的反应之一, 由于同一分子中 存在多种不同性质的 $\mathrm{C}-\mathrm{H}$ 键, 实现 $\mathrm{C}-\mathrm{H}$ 位点选择性 或对映选择性的氧化尤为困难 ${ }^{[2]}$. 同时, 不对称 $\mathrm{C}-\mathrm{H}$ 氧化所形成的手性醇类底物, 极易在相同氧化体系下发 生过度氧化而生成酮，导致手性中心消除(Scheme 1). 尽管 $\mathrm{C}-\mathrm{H}$ 不对称氧化反应的研究已经持续了数十年,
但进展仍然缓慢, 有待深入研究和发展出高效催化剂. 实现 $\mathrm{C}-\mathrm{H}$ 键不对称氧化, 需要深入理解影响 $\mathrm{C}-\mathrm{H}$ 氧 化反应的因素, 从不同的参数和方向调控不对称 $\mathrm{C}-\mathrm{H}$ 氧化反应. 自然界生物酶能活化氧气，形成高价金属氧( $\mathrm{M}-\mathrm{oxo})$ 中间体，可在温和条件下实现 $\mathrm{C}-\mathrm{H} 、 \mathrm{C}=\mathrm{C}$ 的(不对称)氧化反应，是发展高效催化氧化的理想模 型[3]. 本文将综述三十多年来模拟生物酶活性中心结构 发展的小分子金属配合物及其催化的烷烃 C-H 键不对 称氧化反应进展.

\footnotetext{
* Corresponding author. E-mail: wsun@licp.cas.cn

Received June 5, 2020; revised June 19, 2020; published online July 8, 2020.

Dedicated to the 40th anniversary of Chinese Journal of Organic Chemistry.

Project supported by the National Natural Science Foundation of China (Nos. 21773273, 21473226, 21902166), the Key Research Program of Frontier Sciences, CAS (No. QYZDJ-SSW-SLH051) and the Natural Science Foundation of Jiangsu Province (No. BK20170420).

国家自然科学基金(Nos. 21773273, 21473226, 21902166)、中国科学院前沿科学重点研究计划(No. QYZDJ-SSW-SLH051)和江苏省自然科学基金青年 基金(No. BK20170420)资助项目.
} 


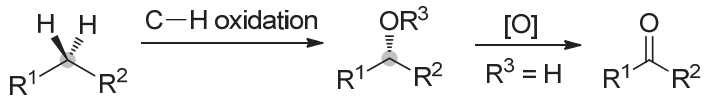

图式 $1 \mathrm{C}\left(\mathrm{sp}^{3}\right)-\mathrm{H}$ 的不对称氧化反应及面临的挑战 Scheme 1 Challenges associated with asymmetric $C\left(\mathrm{sp}^{3}\right)-\mathrm{H}$ oxidation

\section{1 影响 C $\left(\mathrm{sp}^{3}\right)$ - $\mathrm{H}$ 氧化反应的主要因素}

\section{$1.1 \mathrm{C}-\mathrm{H}$ 键的解离能(BDE)大小}

实现 $\mathrm{C}-\mathrm{H}$ 氧化反应的第一步是 $\mathrm{C}-\mathrm{H}$ 键的断裂. 因此, 不同 $\mathrm{C}-\mathrm{H}$ 键的解离能将直接影响 $\mathrm{C}-\mathrm{H}$ 氧化反 应的活性大小 ${ }^{[4]}$. 比如, 苯环 $\mathrm{C}-\mathrm{H}$ 键解离能(BDE)为 $472.6 \mathrm{~kJ} / \mathrm{mol}$, 茮位 $\mathrm{C}-\mathrm{H}$ 键的解离能为 $357.5 \mathrm{~kJ} / \mathrm{mol}$, 三 级 $\mathrm{C}-\mathrm{H}$ 键的解离能为 $400.6 \mathrm{~kJ} / \mathrm{mol}$, 二级 $\mathrm{C}-\mathrm{H}$ 键的解 离能为 $410.6 \mathrm{~kJ} / \mathrm{mol}$, 一级 $\mathrm{C}-\mathrm{H}$ 键的解离能为 422.4 $\mathrm{kJ} / \mathrm{mol}$. 值得注意的是, 一些特殊官能团的 $\alpha$ 位 $\mathrm{C}-\mathrm{H}$ 键 解离能的变化: 比如, 环丙烷 $\alpha$ 位的 $\mathrm{C}-\mathrm{H}$ 键的解离能 为 $407.7 \mathrm{~kJ} / \mathrm{mol}$, 醚类底物氧原子 $\alpha$ 位的 $\mathrm{C}-\mathrm{H}$ 键的解离 能为 $389.3 \mathrm{~kJ} / \mathrm{mol}$, 哌啶中 $\mathrm{N}$ 原子 $\alpha$ 位的 $\mathrm{C}-\mathrm{H}$ 键的解 离能为 $379.7 \mathrm{~kJ} / \mathrm{mol}$. 因此, 从 $\mathrm{C}-\mathrm{H}$ 键的 $\mathrm{BDE}$ 大小考 虑, 不同种类底物 $\mathrm{C}-\mathrm{H}$ 氧化反应发生的活性顺序如 Scheme 2 所示.

\section{2 诱导效应对 $\mathrm{C}-\mathrm{H}$ 氧化反应的影响}

分子中不同位置的 $\mathrm{C}-\mathrm{H}$ 键具有不同的电性, 且由 于氧化剂或者氧化活性中心的亲电性，导致烃类化合物 在发生 $\mathrm{C}-\mathrm{H}$ 氧化反应时具有不同的活性位点. 当分子 中存在一些拉电子基团，比如酯基、氰基、酰亚胺等基 团时, $\mathrm{C}-\mathrm{H}$ 氧化反应往往发生在距离这些拉电子基团 较远的位点. 比如, 1996 年, Asensio 等 $^{[5]}$ 报道了使用甲 基(三氟甲基)-1,3-二环氧丙烷(2, TFDO)为氧化剂氧化 含脂肪链的酯类化合物的 $\mathrm{C}-\mathrm{H}$ 氧化反应(Scheme 3 ). 该研究发现，处于 $\mathbf{1}$ 中酯基远端的亚甲基 $\mathrm{C}-\mathrm{H}$ 被氧化 为酮，而其他亚甲基几乎没有转化. 2007 年, White 等 ${ }^{[6]}$ 也报道了分子结构中既含有三级 $\mathrm{C}-\mathrm{H}$ 也含有酯基的脂 肪链的 $\mathrm{C}-\mathrm{H}$ 氧化反应. 结果显示, 处于酯基远端的三 级 $\mathrm{C}-\mathrm{H}$ 更加容易被氧化，而靠近酯基的三级 $\mathrm{C}-\mathrm{H}$ 则 反应活性更低.

胺和含氮杂环化合物不仅是重要的有机合成中间 体，而且在农药、天然产物以及活性药物分子中广泛存 在. 因此, 含氮化合物的 $\mathrm{C}-\mathrm{H}$ 氧化反应也是 $\mathrm{C}-\mathrm{H}$ 官能 化反应中一个重要的反应类型. 通常情况下, 氮原子 $\alpha$ 位的 $\mathrm{C}-\mathrm{H}$ 键的解离能更小, 会优先发生反应, 比如生

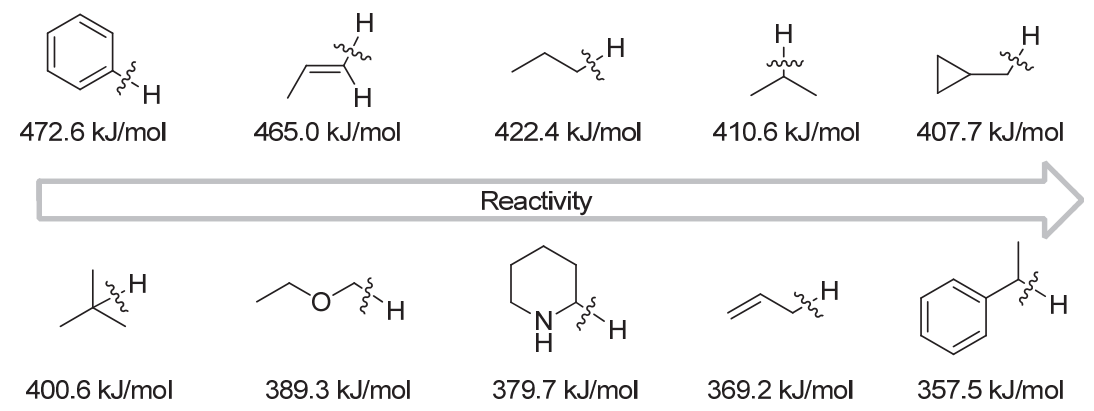

图式 2 不同 $\mathrm{C}-\mathrm{H}$ 键的 $\mathrm{BDE}$ 与 $\mathrm{C}-\mathrm{H}$ 氧化反应活性

Scheme 2 Relationship between $\mathrm{BDE}$ of $\mathrm{C}-\mathrm{H}$ bonds and the reactivity of different $\mathrm{C}-\mathrm{H}$ in oxidation
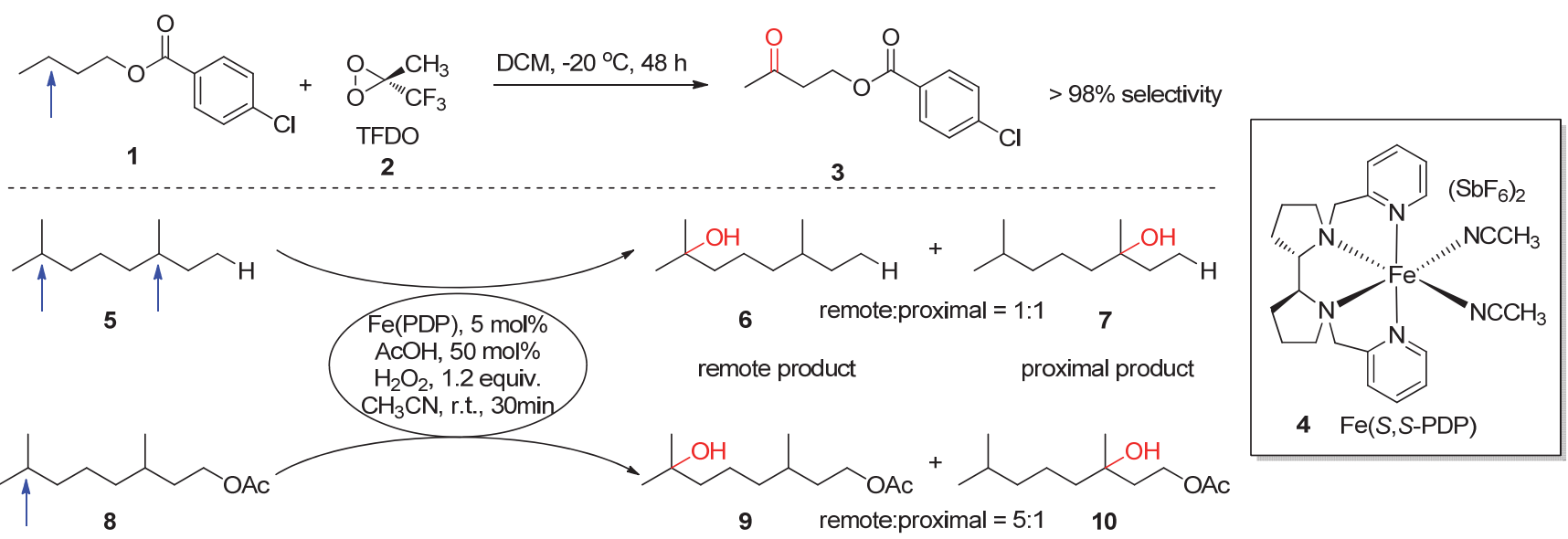

图式 3 诱导效应对 $\mathrm{C}-\mathrm{H}$ 氧化反应位点的影响

Scheme 3 Site-selectivity of $\mathrm{C}-\mathrm{H}$ oxidation influenced by inductive effect 
成亚胺等. 但是如果先将有机胺质子化后形成相应的铵 盐, 翻转含氮基团的电性, 远离氮原子的 $\mathrm{C}-\mathrm{H}$ 键发生 氧化将成为可能. 1993 年, Asensio 等 ${ }^{[7]}$ 就利用四氟厥酸 和正己基胺(11)制备了相应的铵盐 12. 该铵盐在氧化性 条件下转化为相应的 2-甲基四氢吡啶(14). 这一反应中, 在铵盐的诱导下二级 $\mathrm{C}-\mathrm{H}$ 键的氧化发生在远离铵盐的 位点, 生成了相应的酮 13 , 而氨基的 $\alpha$ 位 $\mathrm{C}-\mathrm{H}$ 键没有 发生反应. 随后, 13 发生分子内的脱水反应从而生成 2甲基四氢吡啶(14)(Scheme 4,A). 2015 年, Sanford 等 ${ }^{[8]}$ 使 用相同的策略, 在 1.1 equiv. 硫酸的作用下, 实现了 $N$-乙 基吡咯烷(15)的 $\mathrm{C}-\mathrm{H}$ 氧化反应(Scheme 4, B). 这些实例 也表明了诱导效应在 $\mathrm{C}-\mathrm{H}$ 氧化反应的位点选择性中所 起到的重要作用.

(A)

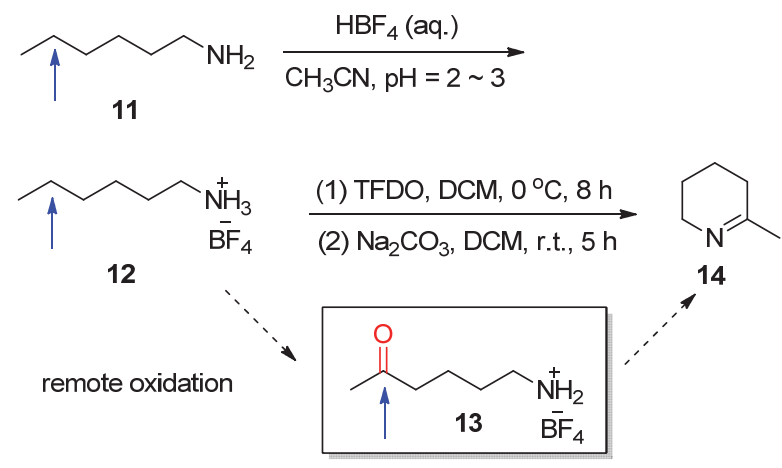

(B)

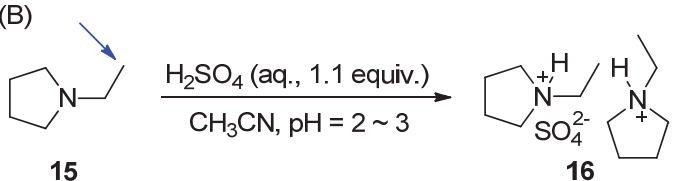

15

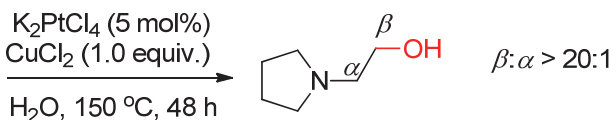

17

图式 4 铵盐诱导的远端 $\mathrm{C}-\mathrm{H}$ 氧化反应

Scheme 4 Remote $\mathrm{C}-\mathrm{H}$ oxidation induced by ammonium

\section{3 导向基团对 $\mathrm{C}$ - $\mathrm{H}$ 氧化反应的影响}

如果分子中存在一种官能团, 其可以改变由电性和 诱导效应等因素控制的 $\mathrm{C}-\mathrm{H}$ 键氧化位点选择性, 则可 将该官能团视为此 $\mathrm{C}-\mathrm{H}$ 氧化反应的导向基团 ${ }^{[9]}$. 理想 的状况是: 这种导向基团在氧化性条件下较为稳定, 而 在完成选择性 $\mathrm{C}-\mathrm{H}$ 氧化后, 还可以在随后的转化反应 中利用起来, 或可以方便地脱除. 2007 年, White 等 $^{[6]}$ 研 究了羧酸作为导向基团控制复杂化合物 $\mathrm{C}-\mathrm{H}$ 氧化位置 选择性的反应(Scheme 5). 他们选取了四氢赤霉酸 18 作 为氧化反应的底物, 发现 $\mathrm{C}-\mathrm{H}$ 氧化发生在与羧基邻近 的二级 $\mathrm{C}-\mathrm{H}$ 键, 生成了相应的内酯 19 , 而不是更加富 电子的三级 $\mathrm{C}-\mathrm{H}$ 键. 同时, 如果将羧酸基团甲酯化以
后，四氢赤霉酸的 $\mathrm{C}-\mathrm{H}$ 氧化反应的转化率将变低下且 产物分布杂乱. 这一例子清楚地证明了 $\mathrm{C}-\mathrm{H}$ 氧化的反 应位点可以由导向基团决定，而不仅仅是由 $\mathrm{C}-\mathrm{H}$ 键的 $\mathrm{BDE}$ 和电性来决定.

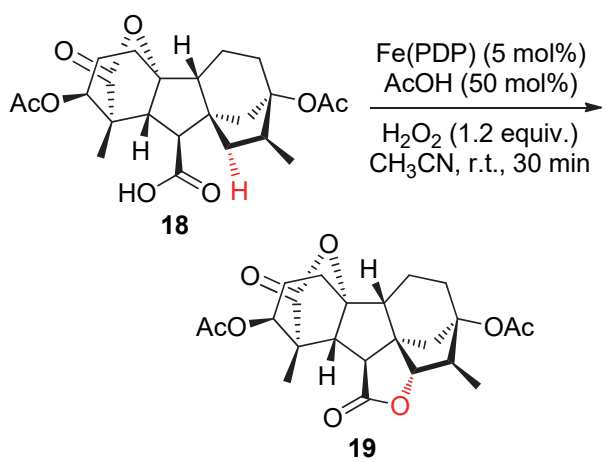

图式 5 羧基作为导向基团对 $\mathrm{C}-\mathrm{H}$ 氧化反应位点的影响 Scheme 5 Site-selectivity of $\mathrm{C}-\mathrm{H}$ oxidation influenced by directing-group

\section{4 位阻因素对 $\mathrm{C}$ 一 $\mathrm{H}$ 氧化反应的影响}

有机分子中与 $\mathrm{C}-\mathrm{H}$ 键相邻或者邻近的位置有大位 阻的基团时，该 $\mathrm{C}-\mathrm{H}$ 键发生氧化的活性和速率就会大 大降低. 如果两个 $\mathrm{C}-\mathrm{H}$ 键的位阻大小差异相对较小, 也可以通过调整催化剂的空间结构来控制含多个类别 的 $\mathrm{C}-\mathrm{H}$ 的氧化反应. 早在 1992 年, Waegell 等 ${ }^{[10]}$ 就发现 了位阻因素对 $\mathrm{C}-\mathrm{H}$ 氧化反应的影响结果(Scheme 6). 柏木烷 20 是一种含有 4 个三级 $\mathrm{C}-\mathrm{H}$ 和 5 个亚甲基的 三环结构的烃类化合物. 他们巧妙地选择柏木烷为底

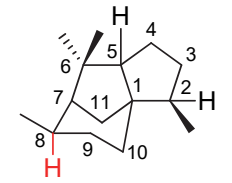

20

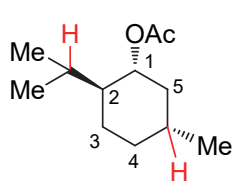

22

$62 \%$ yield, $23: 24=17: 1$

$\mathrm{Fe}(\mathrm{MCPP})(1 \mathrm{~mol} \%)$ $\mathrm{H}_{2} \mathrm{O}_{2}$ (1.2 equiv.) $\underset{\mathrm{CH}_{3} \mathrm{CN}, 0^{\circ} \mathrm{C}, 0.5 \mathrm{~h}}{\stackrel{\mathrm{AcOH}}{(0.5 \text { equiv.) }}}$

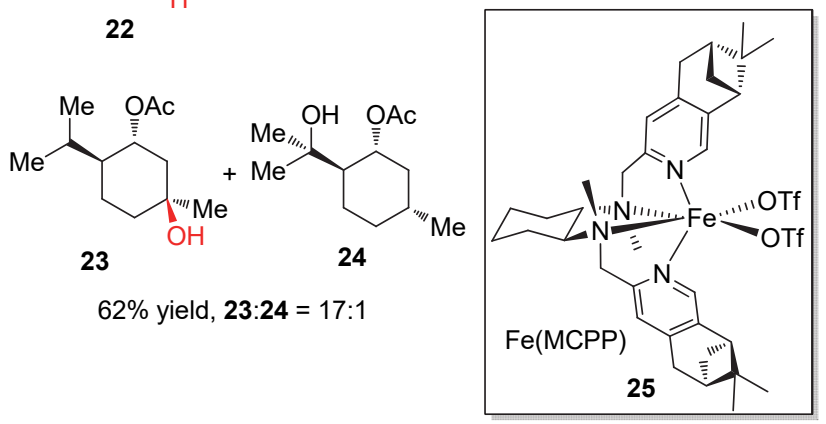

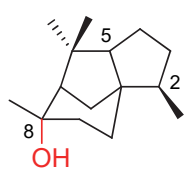

21
图式 6 位阻影响的 $\mathrm{C}-\mathrm{H}$ 氧化反应位点选择性

Scheme 6 Site-selectivity of $\mathrm{C}-\mathrm{H}$ oxidation influenced by steric hindrance 
物, 研究了四氧化钉催化的 $\mathrm{C}-\mathrm{H}$ 氧化, 结果显示: $\mathrm{C}(8)$ 位的三级 $\mathrm{C}-\mathrm{H}$ 键被氧化为叔醇, 而 $\mathrm{C}(2)$ 位和 $\mathrm{C}(5)$ 位的 $\mathrm{C}-\mathrm{H}$ 却没有发生氧化反应. 这一结果正是由于 $\mathrm{C}(2)$ 位 和 C(5)位的邻位有更大的位阻所导致的. 2009 年, Costas 和 Ribas 等 ${ }^{[11]}$ 在研究薄荷醇酯 22 的氧化时, 也发现了类 似的结果. 他们发现 $\mathrm{C}-\mathrm{H}$ 羟化反应发生在位阻较小的 $\mathrm{C}(5)$ 位 $\mathrm{C}-\mathrm{H}$ 而不是位阻较大的 $\mathrm{C}(2)$ 位异丙基的三级 $\mathrm{C}-\mathrm{H}$.

\section{5 环状化合物 1,3-轴向基团导致的张力释放对 C- $\mathrm{H}$ 氧化选择性的影响}

2009 年, Baran 等 ${ }^{[13]}$ 结合 Eschenmoser 和 Schreiber 在 1955 年提出的环张力释放理论和 Houk 等 ${ }^{[12]}$ 的计算结 果, 将甾醇氧化过程中产生选择性的原因一一环张力释 放一一引入到 $\mathrm{C}-\mathrm{H}$ 氧化反应中来, 解释了一些电性极 其相似位阻也接近的多个 $\mathrm{C}-\mathrm{H}$ 键在氧化反应中具有一 定选择性的原因. 他们考察了含有两个三级 $\mathrm{C}-\mathrm{H}$ 的双 环底物在氧化反应中的选择性(Scheme 7). 通过核磁检 测, 发现底物中异丙基中的三级碳的化学位移比与 $\mathrm{H}^{2}$ 相连的碳的化学位移小 $\delta 0.9$, 这也表明异丙基中三级 $\mathrm{C}-\mathrm{H}^{1}$ 要比 $\mathrm{C}-\mathrm{H}^{2}$ 更富电性. 因此, 如果仅仅考虑电性 和位阻的话, $\mathrm{C}-\mathrm{H}$ 氧化反应位点更应倾向发生在 $\mathrm{H}^{1}$ 的 位置. 然而, 实验结果却与之相反: 他们发现即使使用 不同的氧化剂, $\mathrm{C}-\mathrm{H}$ 氧化反应都发生在 $\mathrm{H}^{2}$ 的位置. 这 一现象促使他们考虑是否存在其他因素主导了该类底 物 $\mathrm{C}-\mathrm{H}$ 氧化的位点选择性. 随后, 他们合成了与化合 物 26 结构极其相似的化合物 27. 在这两个化合物中 $\mathrm{H}^{2}$ 的化学位移仅仅相差 $\delta 0.1$. 当把等物质的量的 $\mathbf{2 6}$ 和 $\mathbf{2 7}$ 的混合物在同一反应体系下进行氧化时, 发现化合物 26 被氧化的量是 27 的 3 4 倍. 这一结果进一步证实了: 当底物中有相同电性和位阻的 $\mathrm{C}-\mathrm{H}$ 时, 有利于 1,3 轴 向的环张力释放的 $\mathrm{C}-\mathrm{H}$ 键氧化将优先发生.

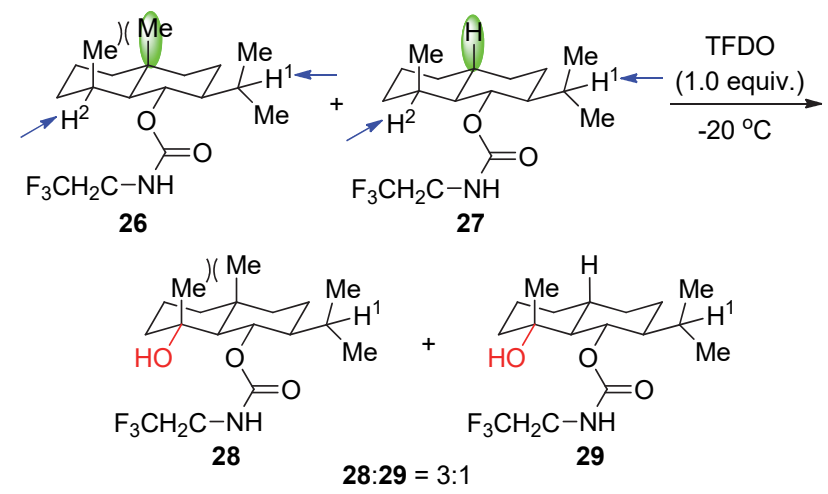

图式 7 环张力释放影响的 $\mathrm{C}-\mathrm{H}$ 氧化反应

Scheme 7 Site-selectivity of $\mathrm{C}-\mathrm{H}$ oxidation influenced by strain release

\section{2 生物酶催化的 $\mathrm{C}\left(s p^{3}\right)-\mathrm{H}$ 氧化反应机理}

在自然界中, 细胞色素 P450 参与了生命体中的甾 醇类激素合成, 是一种末端单加氧酶. 细胞色素 P450的 活性中心结构包含一个血红素铁中心, 在轴向上有一个 半胱氨酸的巯基配体, 轴向的另外一端被水分子占 据 ${ }^{[14]}$. 细胞色素 P450 在催化氧化烷烃时, 烷烃先跟铁 中心结合, 铁配合物即由低自旋态 30 转变成高自旋态 31, 然后被还原为二价 32, 接着被氧气氧化生成三价过 氧自由基物种 33 , 进一步通过质子偶合电子转移过程 生成三价 $\mathrm{Fe}(\mathrm{III})-\mathrm{OOH}$ 物种 34; 随后, $\mathrm{Fe}(\mathrm{III})-\mathrm{OOH}$ 与质 子结合释放出一分子水后形成五价 $\mathrm{Fe}(\mathrm{V})=\mathrm{O}$ 物种 $\mathbf{3 5}$, 35 为真正的氧化性中间体. 烷烃被氧化时, $\mathrm{C}-\mathrm{H}$ 键的 断裂和 $\mathrm{C}-\mathrm{O}$ 键的生成, 可能是协同过程, 也可能是摘 氢一氧反馈的分步过程, 而五价 $\mathrm{Fe}(\mathrm{V})=\mathrm{O}$ 物种最终变成 三价 $\mathrm{Fe}(\mathrm{III})$ 物种进入下一次催化循环(Scheme 8).

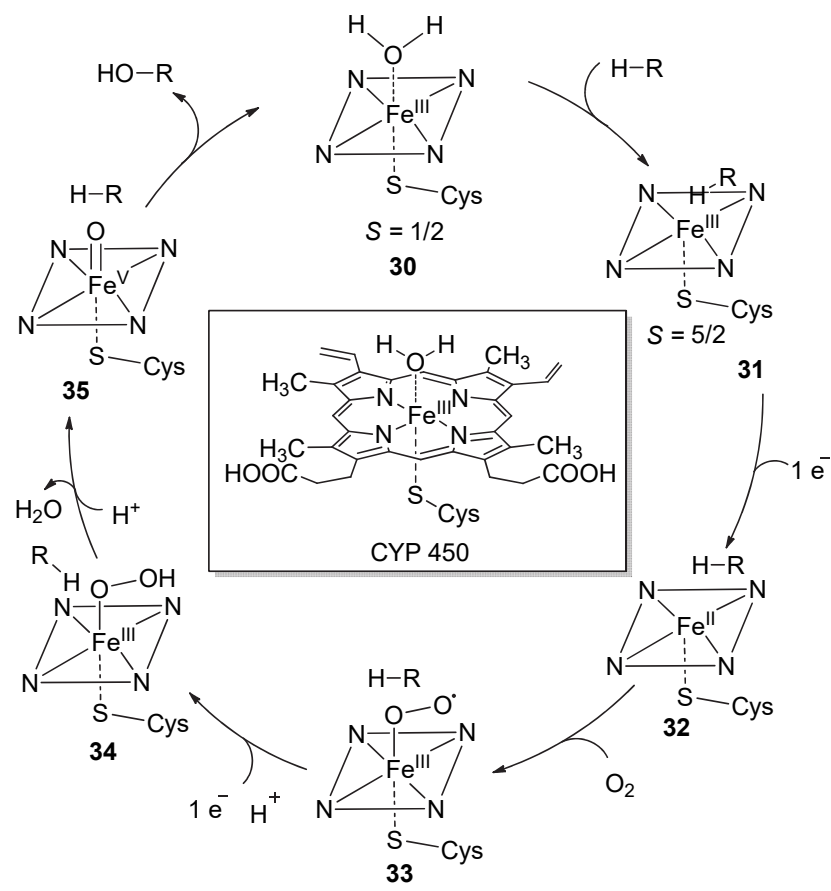

图式 8 细胞色素 P450 催化的烷烃羟化反应机理 Scheme 8 Mechanism of hydroxylation catalyzed by CYP 450

师法自然, 化学家能够从生物酶催化的氧化反应受 到启发而发展出高效的催化氧化反应新体系. 目前，针 对 $\mathrm{C}-\mathrm{H}$ 键不对称氧化反应发展的小分子金属配合物仿 生催化剂主要有两大类: 血红素酶模拟物卟啉金属配合 物及基于非血红素金属蛋白酶的手性多齿氮配体的金 属配合物 ${ }^{[15]}$.

\section{3 卟啉金属配合物催化的 $\mathrm{C}\left(\mathrm{sp}^{3}\right)-\mathrm{H}$ 键不对称 氧化反应}

1989 年, Groves 等 ${ }^{[16]}$ 结合细胞色素 P450 模型化合 
物在烯烃不对称环氧化反应中的催化机制, 设计并合成 了一种新型的四苯基卟啉配体，并首次报道了卟啉 $\mathrm{Fe}$ 、 $\mathrm{Mn}$ 配合物催化 $\mathrm{C}\left(\mathrm{sp}^{3}\right)-\mathrm{H}$ 不对称羟化反应. 他们将手性 联菜引入四苯基卟啉骨架, 用联芸作为桥联基团, 制备 出了一种新型的手性卟啉配体并分别合成了相应的 Fe、 Mn 配合物 (Scheme 9). 使用碘氧苯为氧化剂时, 他们分 别考察了铁和锰配合物在乙苯 $\mathrm{C}-\mathrm{H}$ 差化反应中的催化 性能, 发现二者的催化活性和选择性均不同: 铁配合物 催化时, 产率为 $40 \%$, 对映选择性为 $40 \%$, 产物的醇酮 比为 $2.3: 1$; 使用锰配合物催化该反应, 产率更高为 $77 \%$, 对映选择性却更差 $(26 \%$ ee $)$, 产物的醇酮比为 $1.3 ： 1.1999$ 年, Gross 等 ${ }^{[17]}$ 利用缩酮修饰了四苯基卟啉 骨架，合成了手性卟啉钉配合物 43. 与之前工作不同的 是, 他们采用 2,6-二氯吡啶氮氧化物 42 为氧化剂, 尝试 了三级苄位 $\mathrm{C}-\mathrm{H}$ 的羟化反应, 此反应优点是醇产物不 会被进一步氧化为酮. 他们发现, 在室温条件下 2-苯基 丁烷(40) 以 $54 \%$ 的产率和 $27 \%$ 的 $e e$ 被氧化成醇 41 . 当降 低反应温度到 $10{ }^{\circ} \mathrm{C}$ 时反应的产率降低到 $23 \%$, 反应的 对映选择性升高到 38\% (Scheme 10). 同年, 支志明课题 组 ${ }^{[18]}$ 利用 Halterman 发展的氢化蒽骨架修饰的卟啉配体, 制备出相应的钉配合物 $\mathbf{4 5}$, 考察了其在苄位 $\mathrm{C}-\mathrm{H}$ 氧化 反应中的催化性能. 他们同样使用 2,6-二氯吡啶氮氧化 物为氧化剂. 在二价钉配合物的催化下，乙苯被以 $62 \%$ 的产率转化为醇, 其对映选择性为 $72 \%$, 而醇酮比为 $1.67: 1$ (Scheme 11).

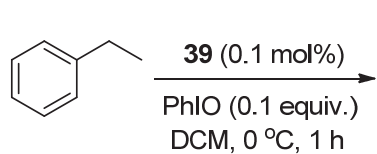

36

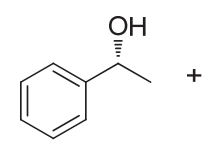

37

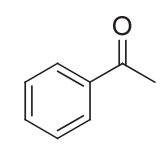

38

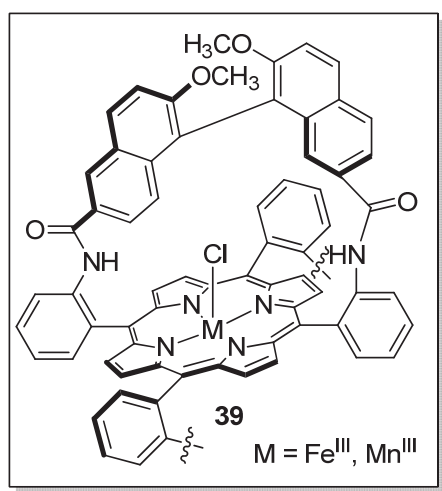

$\mathrm{M}=\mathrm{Fe}: 40 \%$ yield, $40 \%$ ee, $\mathrm{A} / \mathrm{K}=2.3: 1$

$M=M n: 77 \%$ yield, $26 \%$ ee, $A / K=1.3: 1$

图式 9 手性联䒺桥连的卟啉配合物在乙苯 $\mathrm{C}-\mathrm{H}$ 氧化反应中 的催化性能

Scheme 9 Asymmetric $\mathrm{C}-\mathrm{H}$ oxidation catalyzed by porphyrin complex modified using binaphthyl skeleton
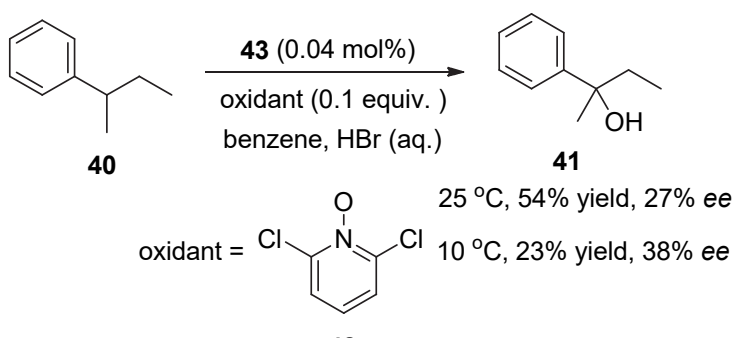

42

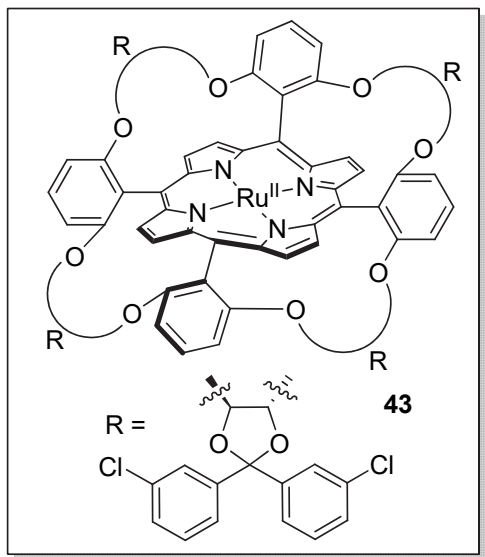

图式 10 手性缩酮桥连的卟啉钉配合物催化的苄位 $\mathrm{C}-\mathrm{H}$ 氧 化反应

Scheme 10 Asymmetric C-H oxidation catalyzed by porphyrin complex bridged using ketal
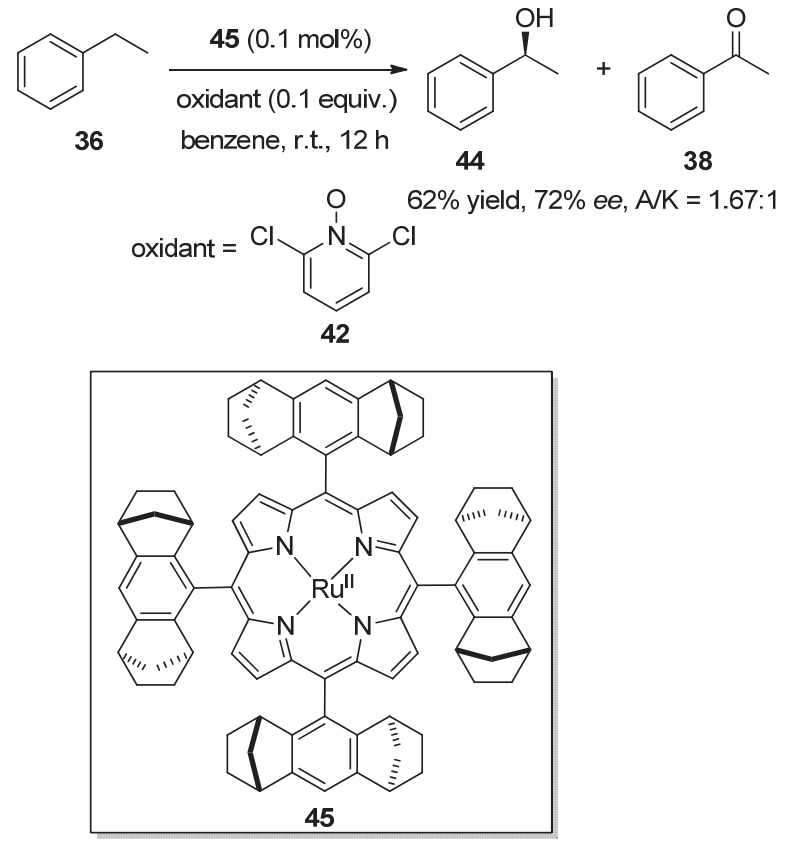

图式 11 Halterman 卟啉配体及其钉配合物催化的苄位 $\mathrm{C}-\mathrm{H}$ 氧化反应

Scheme 11 Asymmetric benzylic C-H oxidation catalyzed by porphyrin-Ru complex

2012 年, Simonneaux 等 ${ }^{[19]}$ 进一步将 Halterman 卟啉 配体进行优化, 引入了四个磺酸基, 合成了水溶性的卟 
啉锰配合物 46 (Scheme 12). 在以双氧水为氧化剂的条 件下, 乙苯的苄位 $\mathrm{C}-\mathrm{H}$ 氧化反应得以实现. 产物中醇 酮比例为 $1.3: 1$, 醇产物的 $e e$ 为 $38 \%$. 随后, 他们使用

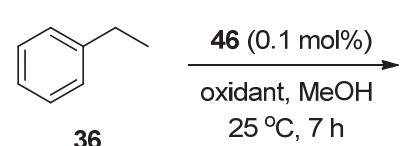

$\mathrm{H}_{2} \mathrm{O}_{2}$ (5.0 equiv.)

$\mathrm{Phl}(\mathrm{OAc})_{2}$ (0.1 equiv.)

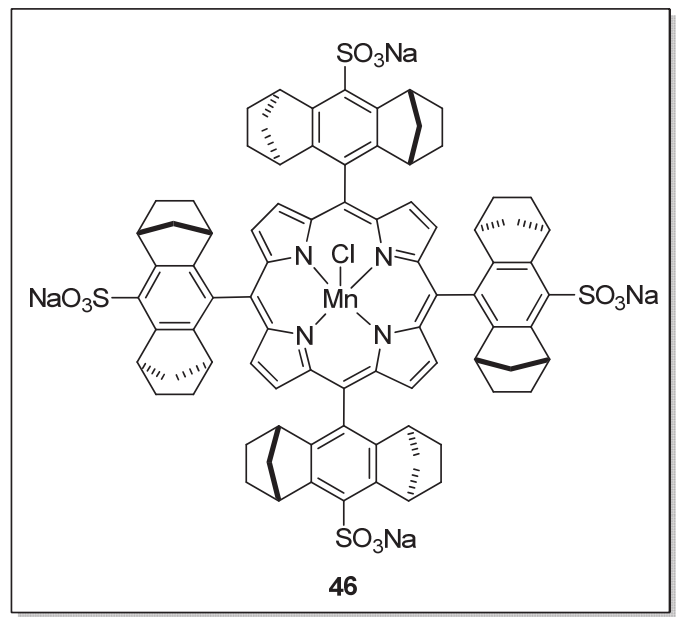

图式 12 水溶性卟啉锰配合物催化的乙苯 $\mathrm{C}-\mathrm{H}$ 氧化反应 Scheme 12 Asymmetric benzylic $\mathrm{C}-\mathrm{H}$ oxidation catalyzed by water-soluble porphyrin-Mn complex
二醋酸碘苯作为氧化剂, 产物的醇酮比最高为 $91: 9$ 且 产物手性醇的对映选择性最高达到 $75 \% e e^{[20]}$.

由于手性卟啉配体合成比较复杂, 随后的很多年此 领域进展缓慢. 2015 年, Bach 课题组 ${ }^{[21]}$ 将氢键导向的反 应概念引入到螺环氧化吲哚的 $\mathrm{C}-\mathrm{H}$ 氧化反应中. 通过 去对称化的策略, 实现了螺环氧化吲哚的对映选择性 $\mathrm{C}-\mathrm{H}$ 氧化反应. 在此之前, 他们 ${ }^{[22]}$ 通过 Sonogashira 反 应在卟啉配体中引入刚性大位阻手性酰胺骨架, 并以其 新型的含酰胺导向基团的卟啉钓配合物成功实现了烯 烃的不对称环氧化反应. 作者发现, 此手性 $\mathrm{Ru}$ 催化剂 49 催化螺环氧化吲哚 47 的 $\mathrm{C}-\mathrm{H}$ 键氧化的中间产物醇 不稳定，容易发生逆 aldol 反应，导致反应的产率较低、 对映选择性极不稳定. 因此, 在完成 $\mathrm{C}-\mathrm{H}$ 氧化反应后, 又将反应混合液置于 Swern 氧化或者氯铬酸吡啶(PCC) 氧化的条件下继续氧化, 将羟基产物转化为酮. 这一改 进大大提高了产物手性酤 48 的收率, 且反应的对映选 择性也基本保持(Scheme 13, A). 2018 年, 该课题组 ${ }^{[23]}$ 利 用相似的策略, 实现了 3,3-二甲基-3,4-二氢喹诺酮(50) 的茮位 $\mathrm{C}-\mathrm{H}$ 氧化反应(Scheme 13, B). 与螺环氧化吲哚 的茮位 $\mathrm{C}-\mathrm{H}$ 氧化不同的是, 此反应使用碘氧苯为氧化 剂, 且产物停留在醇产物. 最近, 他们又将该反应体系 应用到 3-苯亚甲基喹诺酮(51)的苄位 $\mathrm{C}-\mathrm{H}$ 不对称羟化 反应中(Scheme 13, C), 该反应的对映选择性可高达 $99 \%{ }^{[24]}$.
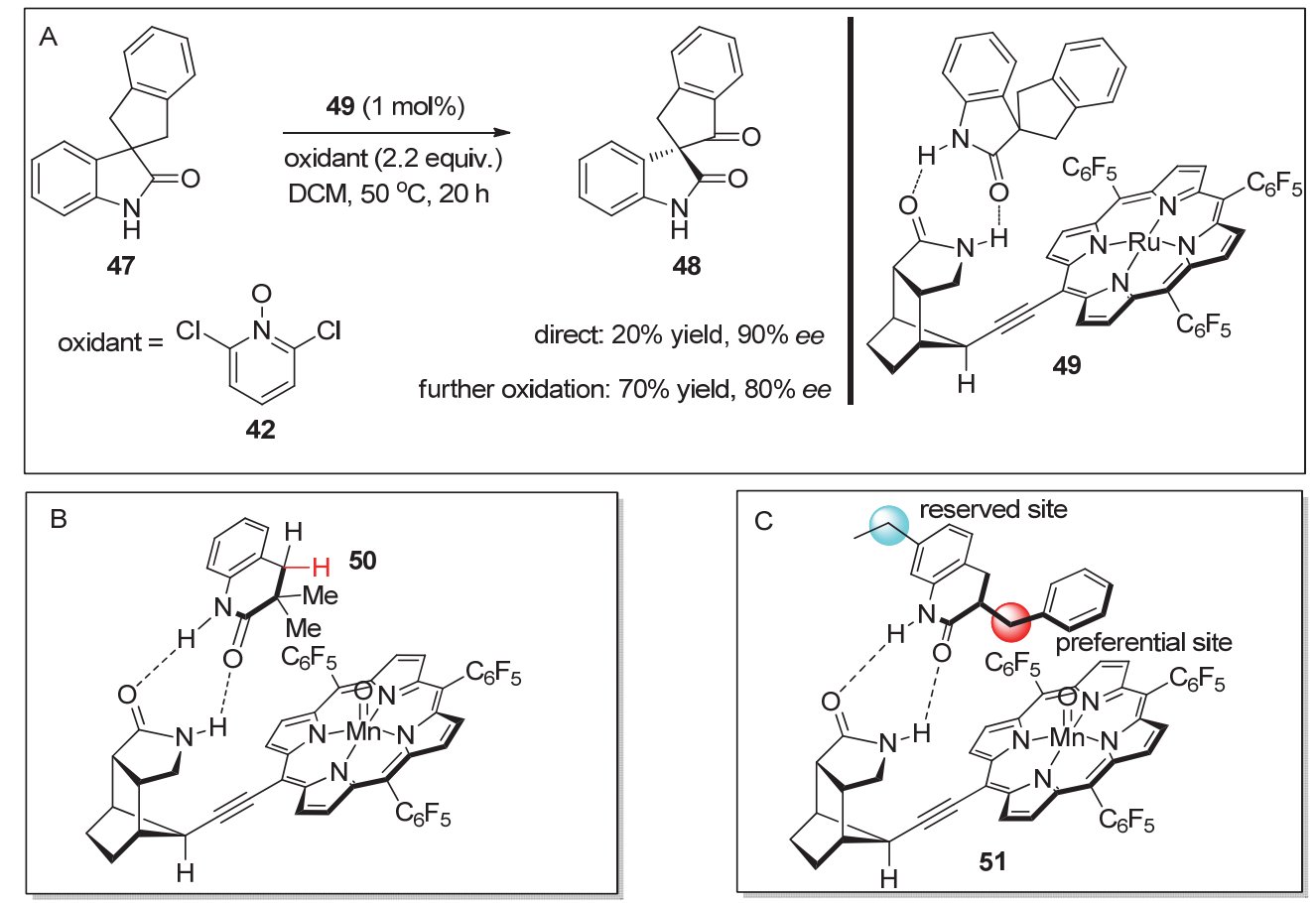

图式 13 刚性酰胺骨架修饰的卟啉配合物催化的 $\mathrm{C}-\mathrm{H}$ 氧化反应

Scheme 13 Asymmetric $\mathrm{C}-\mathrm{H}$ oxidation catalyzed by porphyrin complex modified using amide 


\section{4 手性 salen 金属配合物催化的 $\mathrm{C}\left(\mathrm{sp}^{3}\right)$ - $\mathrm{H}$ 不 对称氧化反应}

手性 salen 配体是 20 世纪 90 年代发展起来的一类 手性配体, 其在众多的不对称反应中得到应用, 被认为 是一类优势配体 ${ }^{[25]}$. Salen 金属配合物为 N2-O2 型配体, 其金属配合物的 N2-O2 配位原子通常在同一平面，通常 被认为是一种类卟啉配体. 由于 salen 金属配合物在烯 烃的不对称环氧化反应中表现出的优异的催化性能, 自 然也被考虑用于 $\mathrm{C}$ - $\mathrm{H}$ 不对称氧化反应

1996 年, Katsuki 课题组 ${ }^{[26]}$ 将轴手性联菜骨架的 salen-Mn 配合物应用到苄位 $\mathrm{C}-\mathrm{H}$ 差弪化反应中. 他们通 过与 Jacobsen 催化剂作比较, 发现在 Salen 配体中引入 的轴手性联菜骨架才是真正有利于不对称 $\mathrm{C}-\mathrm{H}$ 羟化反 应手性诱导的决定因素. 同样的, 以乙苯的氧化为例, 在该体系的标准条件下, 乙苯以 $22 \%$ 的产率和 $53 \%$ ee 转化为 1-苯基乙醇(Scheme 14, A). 1998 年，他们又通过 对手性二胺和联菜骨架的手性匹配笁选, 调整了氧化 剂、反应溶剂和温度, 最终实现了四氢呋喃类似物 ${ }^{[27]}$ 和 吡咯烷类底物 ${ }^{[28]}$ 的去对称 $\mathrm{C}-\mathrm{H}$ 羟化反应(Scheme 14, $\mathrm{B}, \mathrm{C})$. 这是第一例金属配合物催化的内消旋底物的去 对称 $\mathrm{C}-\mathrm{H}$ 羟化反应研究.

A

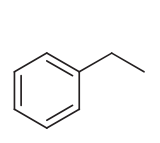

36

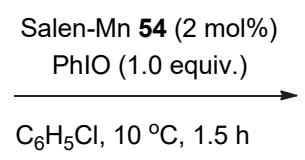

$\mathrm{C}_{6} \mathrm{H}_{5} \mathrm{Cl}, 10^{\circ} \mathrm{C}, 1.5 \mathrm{~h}$

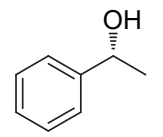

37

$22 \%$ yield, $53 \%$ ee

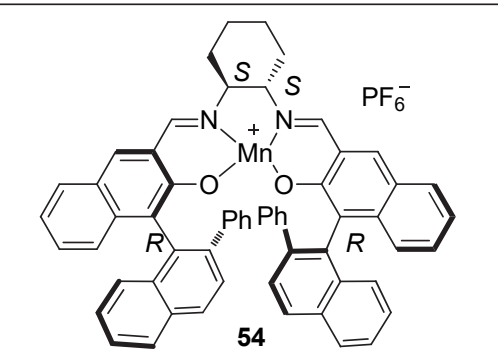

Murahashi 课题组 ${ }^{[29]}$ 于 2004 年使用轴手性联菜骨架 将 salen 配体桥连起来, 考察了其在狮满 60 的苄位 C$\mathrm{H}$ 氧化反应中的催化性能(Scheme 15). 作者还研究了反 应体系中的添加剂对反应结果的影响. 他们发现 1,5-二 环已基咪唑(62)能提高反应的产率和对映选择性，且该 反应产物的醇酮比例也得到了一定程度的提高. 此外, 该课题组 ${ }^{[30]}$ 尝试了 salen-Mn 配合物在 $\mathrm{C}\left(\mathrm{sp}^{3}\right)-\mathrm{H}$ 的去对 称氧化反应中的催化性能. 除了使用碘氧苯为氧化剂 外，在该体系中，他们还添加了 0.5 equiv.的 4-苯基吡啶 氮氧化物 66 来提高反应的活性和选择性. 尽管通过去 对称化的策略，2-甲基-1,3-二苯基丙烷(64)可以转化为 手性酮 65, 但是反应的产率和对映选择性都不能令人 满意.

\section{5 非血红素金属配合物一一手性四齿氮配合物 催化的 $\mathrm{C}\left(\mathrm{sp}^{3}\right)-\mathrm{H}$ 不对称氧化反应}

在过去几十年，模拟氧化酶的活性中心结构，设 计、合成新型手性配体及其金属配合物，实现化学选择、 位点选择、对映选择地简单烃 $\mathrm{C}-\mathrm{H}$ 的氧化反应一直是 有机化学家追求的目标. 除了卟啉骨架和类卟啉 salen 骨架，选用不同的手性胺骨架以及氮供体，调节配体的
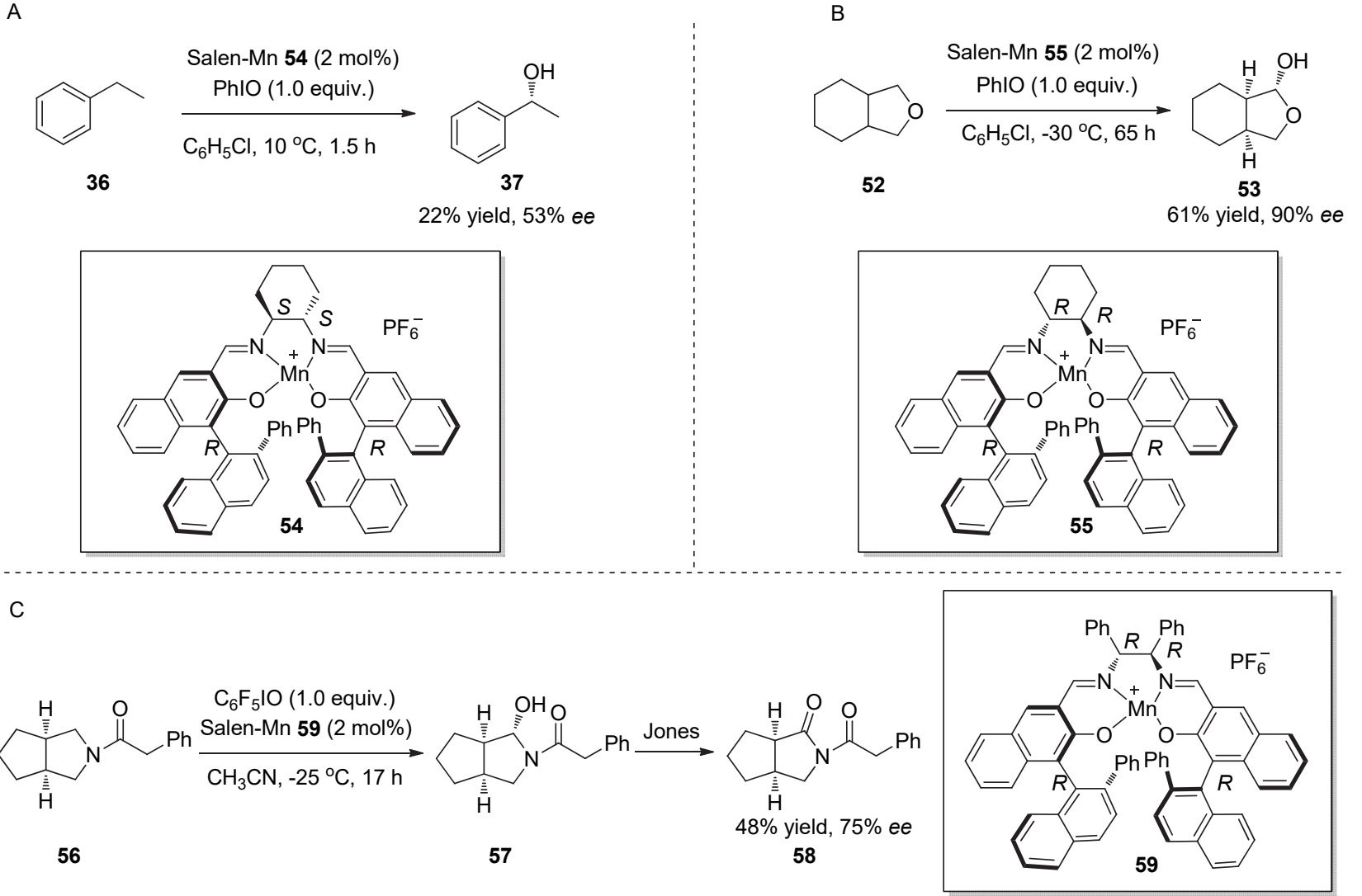

图式 14 联萗骨架修饰的 Salen-Mn 配合物催化的不对称 C-H 羟化反应

Scheme 14 Asymmetric C-H hydroxylation catalyzed by salen-Mn complex modified using binaphthyl skeleton 


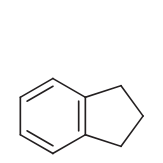

60

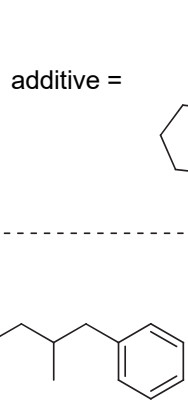

64<smiles>[O-][n+]1ccc(-c2ccccc2)cc1</smiles>
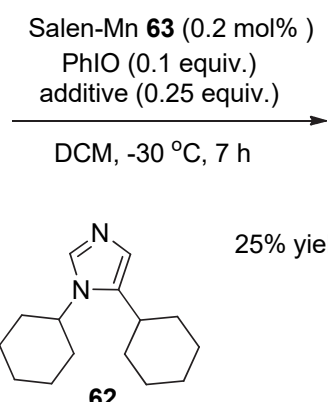

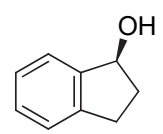

61

$25 \%$ yield, $34 \%$ ee, $A / K=4.1: 1$

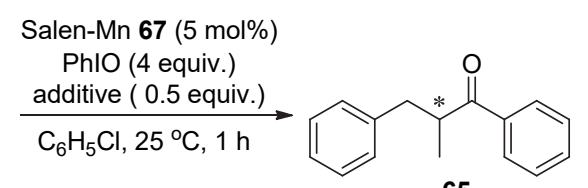

65

$24 \%$ yield, $22 \%$ ee
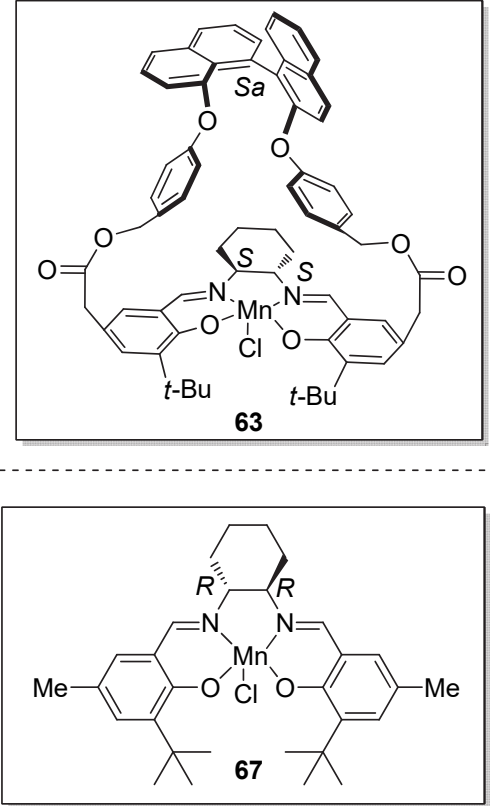

图式 15 联菜骨架桥连的 salen-Mn 配合物在不对称 $\mathrm{C}-\mathrm{H}$ 氧化反应中的催化性能

Scheme 15 Asymmetric C $-\mathrm{H}$ oxidation catalyzed by salen-Mn complex bridged using binaphthol

空间结构和电性，进而控制金属配合物的催化活性和选 择性是科学家们一致采用的策略. 近十多以来, 基于手 性二胺与吡啶等杂环构建的线性四氮配体所发展的铁、 锰配合物, 作为非血红素酶的模型化合物, 在不对称氧 化反应中表现出优秀的催化活性和对映选择性.

2017 年, Costas 课题组 ${ }^{[31]}$ 将前期发展的新型配 体一一使用手性环己二胺为手性骨架, 三异丙基硅基修 饰的吡啶为氮供体一一应用到烷烃的不对称 $\mathrm{C}-\mathrm{H}$ 氧化 反应中, 获得了优异的反应结果. 这一反应采用了去对 称化的策略, 当使用酰胺为导向基团时, 环已烷的 $\mathrm{C}$ $\mathrm{H}$ 氧化发生在 $\mathrm{C}(3)$ 位而不是 $\mathrm{C}(4)$ 位 (Scheme 16, A). 特别 是当使用特戊酰胺为导向基团, 环丙烷甲酸为添加剂 时, $\mathrm{C}(3)$ 位氧化成酮的产率为 $90 \%$, 对映选择性为 $90 \%$. 对于非血红素 $\mathrm{N} 4$ 金属配合物/ $\mathrm{H}_{2} \mathrm{O}_{2}$ 催化氧化反应体系 中, 有机羧酸添加剂是决定反应活性和选择性的重要因 素 ${ }^{[32]} .2018$ 年, 我们课题组 ${ }^{[33]}$ 以环氧化反应为模板, 深 入研究了酸添加剂在不对称氧化反应中的作用: 双氧水 氧化锰配合物形成可能的 Mn-OOH 物种, 然后有机羧 酸辅助此物种的 $\mathrm{O}-\mathrm{O}$ 键异裂, 进而脱去一分子水形成 $\mathrm{Mn}=\mathrm{O}$ 活性中间体; 同时, 酸配位在金属中心, 有效提 高中间体的不对称诱导能力, 大位阻的羧酸将有助于提 高反应的对映选择性.

此外, Costas 课题组 ${ }^{[34]}$ 还尝试了 1,4-二取代的环己 烷的 C-H氧化. 结果表明: 1,4-顺式取代的底物 71 中一 个酰胺基团被氧化为羰基, 而另一个酰胺基团保留; 1,4-反式取代的底物 72 则不能发生反应(Scheme 16, B).
这一结果提供了一种双取代环已烷的拆分策略. 从另外 一方面说, 也恰巧证明了环状结构烷烃 $\mathrm{C}-\mathrm{H}$ 氧化反应 中的 1,3-轴向的张力释放因素对 $\mathrm{C}-\mathrm{H}$ 氧化反应位点的 影响.

Bryliakov 课题组 ${ }^{[35]}$ 在同年报道了乙苯类苄位 $\mathrm{C}-\mathrm{H}$ 氧化的反应结果. 他们使用三氟乙氧基修饰的四齿氮配 合物 76 为催化剂, 其中手性二胺骨架为联吡咯(Scheme 17). 为了提高反应产物的醇酮比例, 他们使用了 0.35 equiv. 的 $\mathrm{H}_{2} \mathrm{O}_{2}$ 作氧化剂, 使用 Boc- $L$-脯氨酸为添加剂. 然而反应的结果并不令人满意: 乙苯的转化率仅仅为 $31 \%$, 产物苯乙醇和苯乙酮的比例为 $1: 1.2$, 苯乙醇的 对映选择性为 $76 \%$. 这一反应结果说明: 在四齿氮金属 配合物为催化剂, 双氧水为终端氧化剂, 酸为添加剂的 反应体系中, $\mathrm{C}-\mathrm{H}$ 氧化反应想要停留在手性醇的阶段 是非常难以控制的.

我们课题组 ${ }^{[36]}$ 在过去的十多年中也一直致力于高 效不对称氧化体系的构建, 在模拟非血红素蛋白酶活性 中心结构的过程中, 发展了一系列易于制备、性能优异 的四齿氮配体及其金属配合物. 这些配合物在烯烃环氧 化反应中展现出了较高的催化活性和不对称诱导能 力 $^{[37]}$. 鉴于这些研究基础, 我们尝试挑战烷烃 $\mathrm{C}-\mathrm{H}$ 键 的不对称氧化反应. 设计了一类具有螺环结构的化合物 77 , 经过催化剂的系统篮选, 发现 $L$-脯氨酸衍生的手性 配体的锰配合物 $82(S-$ peb-Mn) 在此类螺环底物 $\mathrm{C}-\mathrm{H}$ 氧 化反应中展现出优良的催化性能(Scheme 18). 六元螺 环化合物氧化产物的对映选择性可达 $94 \%$, 五元螺环的 
A

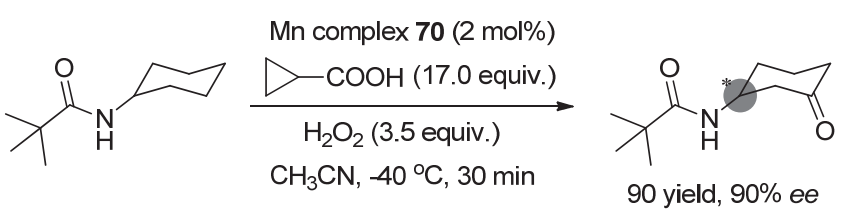

68

69
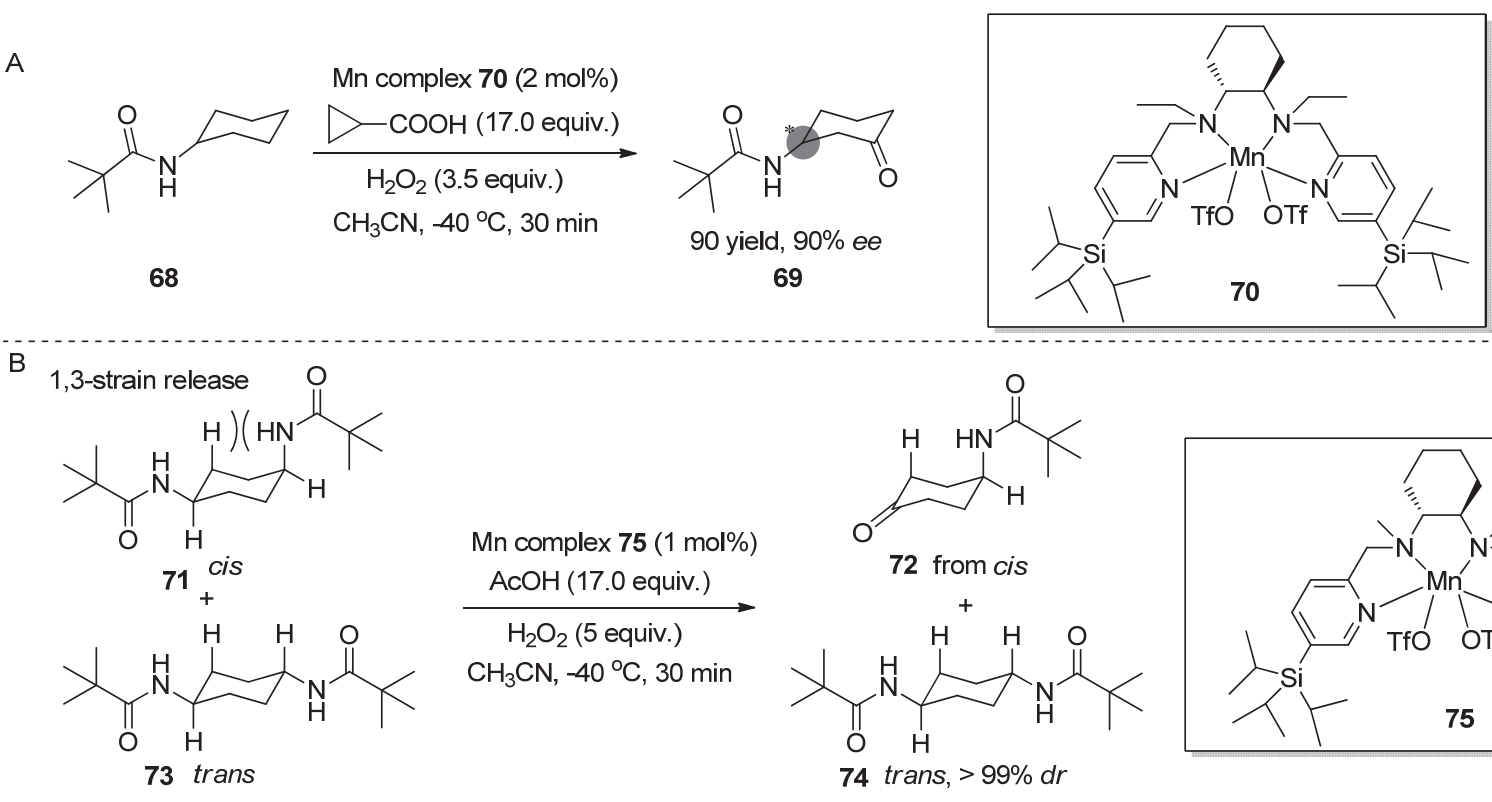<smiles></smiles>

72 from cis
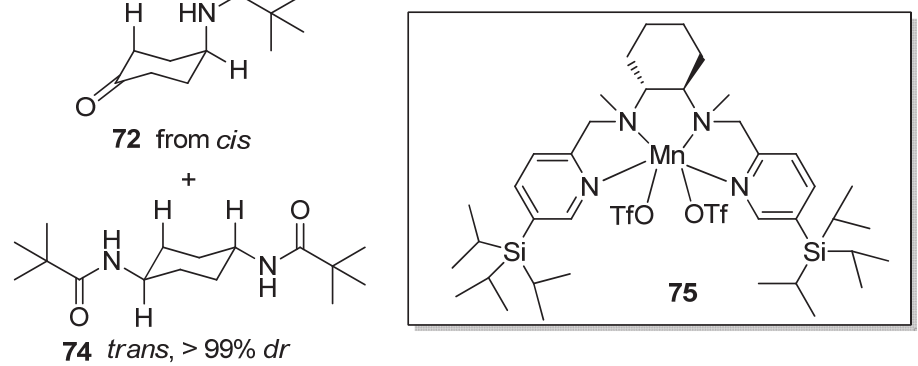

图式 16 三异丙基硅基修饰的手性环己二胺骨架四齿氮配合物催化的 $\mathrm{C}-\mathrm{H}$ 氧化反应

Scheme 16 Asymmetric C $-\mathrm{H}$ oxidation catalyzed by tetradentate nitrogen complex modified using tri-isopropylsilyl group

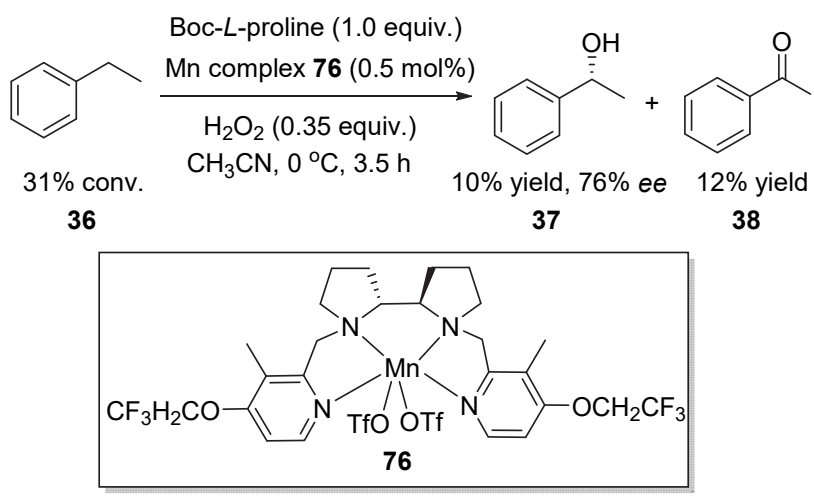

图式 17 三氟乙氧基修饰的手性联吡咯骨架四齿氮配合物催 化的 $\mathrm{C}-\mathrm{H}$ 氧化反应

Scheme 17 Asymmetric C-H oxidation catalyzed by tetradentate nitrogen complex modified using trifluoroethoxyl group

$\mathrm{C}-\mathrm{H}$ 氧化产物的对映选择性可达 $98 \%$. 值得一提的是, 尽管该类底物中, 有多个苠位 $\mathrm{C}-\mathrm{H}$, 但是该反应仍然 给出了优异的位点选择性和对映选择性 ${ }^{[38]}$. 此外, 该反 应与之前报道的茮位 $\mathrm{C}-\mathrm{H}$ 氧化反应的产率相比, 同样 有很大程度的提高. 随后, 我们还进一步研究了含氮杂 环的螺环化合物的 $\mathrm{C}-\mathrm{H}$ 氧化反应. 比如, 我们研究了 在药物活性分子和天然产物中较为常见的螺环氧化吲 哚骨架和螺环喹啉酮骨架的 $\mathrm{C}-\mathrm{H}$ 氧化 ${ }^{[39]}$. 结果显示: 不管是螺环氧化吲哚还是螺环喹啉酮底物, 都能被很好 地转化为相应的手性酮; 当降低反应的温度和氧化剂的 用量时，该反应以 $22 \% \sim 41 \%$ 的产率获得了螺环喹啉酮 的 C-H 差弪化产物, 对映选择性高达 $99 \%$.

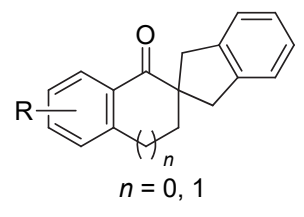

77<smiles>[R][X]c1ccccc1C(=O)[C@]12Cc3ccccc3C(=O)[C@]1(C)C2</smiles>

up to $94 \%$ yield, $98 \%$ ee

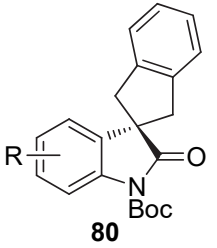

up to $94 \%$ ee

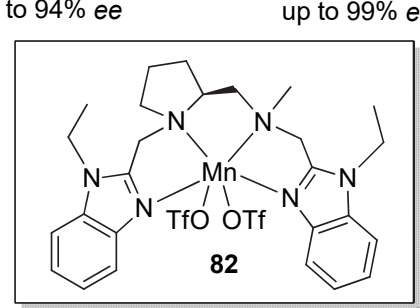

图式 18 脯氨酸衍生的 $S$-peb-Mn 配合物催化的 C-H 氧化反 应

Scheme 18 Asymmetric C-H oxidation catalyzed by $S$-pebMn complex derived from $L$-proline

金刚烷骨架中含有多个亚甲基以及三级 $\mathrm{C}-\mathrm{H}$, 因 
此成为研究 $\mathrm{C}-\mathrm{H}$ 氧化反应的模板底物之一. 最近, Costas 和 Bietti 等 ${ }^{[40]}$ 利用羧酸作为导向基, 实现了金刚 烷骨架的定点 $\mathrm{C}-\mathrm{H}$ 氧化反应，合成了内酯化产物 $\mathbf{8 4}$, 反应的产率可达 $88 \%$, 对映选择性最高可以达到 $99 \%$ (Scheme 19). 在该反应中, $\mathrm{C}-\mathrm{H}$ 氧化发生在羧基 $\gamma$ 位, 这一位置远离拉电子基团, 适合形成五元环内酯产物. 值得注意的是, 在羧基的诱导下更富电子的三级 $\mathrm{C}-\mathrm{H}$ 并没有发生氧化反应，且当羧基 $\gamma$ 位附近有取代基形成 位阻时, 可以通过催化剂结构的调控实现 $\mathrm{C}-\mathrm{H}$ 氧化位 点的控制.

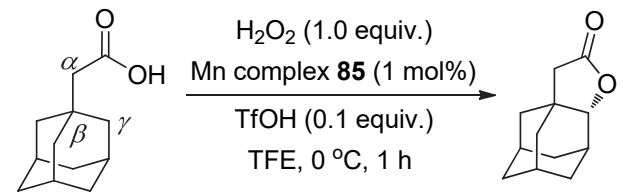

83 $68 \%$ yield, $98 \%$ ee 84

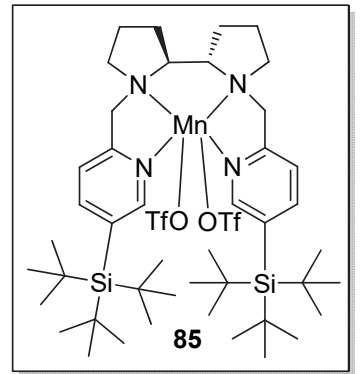

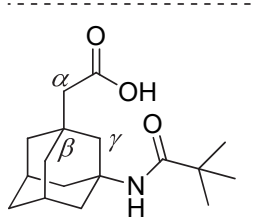

87

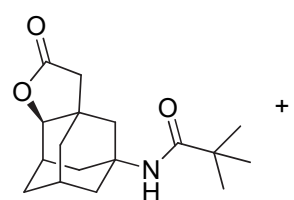

89
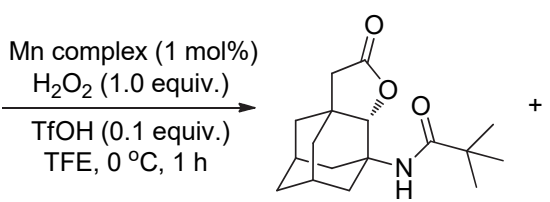

88

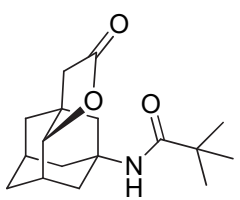

90

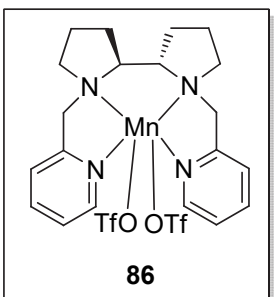

Mn-PDP 86:

$\mathbf{8 8 / 8 9 / 9 0}=1 / 3 / 6$

Mn- ${ }^{\text {TIBSPDP } 85}$

$\mathbf{8 8 / 8 9} / \mathbf{9 0}=1 / 7.4 / 2.5$

图式 19 三异丁基硅基修饰的 Mn-PDP 配合物催化的金刚烷 $\mathrm{C}$ - H 氧化反应

Scheme $19 \mathrm{C}-\mathrm{H}$ Oxidation of adamantane catalyzed by Mn-PDP complex modified using tri-isobutylsilane

\section{6 结论与展望}

$\mathrm{C}$ - $\mathrm{H}$ 键广泛存在于有机分子中, 其氧化反应可直 接向分子中引入氧或者羰基官能团, 或者在复杂分子全 合成的后期实现直接官能化，是现代有机合成中一类重 要的反应. 特别是一些简单烃类化合物的 $\mathrm{C}-\mathrm{H}$ 不对称 氧化反应将为生物活性分子的合成提供新的合成策略, 提高复杂分子全合成的效率. 然而，也正是由于同一有 机分子中存在多个 $\mathrm{C}-\mathrm{H}$ 键, 并且 $\mathrm{C}-\mathrm{H}$ 之间的立体性 差异较小, 难以识别, 给简单烷烃 $\mathrm{C}-\mathrm{H}$ 的不对称氧化 提出了更高的要求. 本文综述了模拟生物酶发展的一系 列新型手性卟啉、四氮配体金属配合物催化 $\mathrm{C}-\mathrm{H}$ 键不 对称氧化反应的研究进展, 并结合影响 $\mathrm{C}\left(\mathrm{sp}^{3}\right)-\mathrm{H}$ 的氧 化反应的因素、模拟酶金属配合物催化的 $\mathrm{C}-\mathrm{H}$ 氧化反 应的机理，从配体类型的角度阐述了当前所实现的 $\mathrm{C}-$ $\mathrm{H}$ 键不对称氧化的一些实例. 尽管目前这些研究仅仅是 针对一些特殊底物取得了较高的催化活性和立体控制 能力, 但是这些结果为人们发展更加高效和实用的不对 称 $\mathrm{C}-\mathrm{H}$ 氧化催化新体系奠定了坚实的基础，提供了一 些催化剂设计的思路. 我们也期待, 随着对 $\mathrm{C}-\mathrm{H}$ 不对 称氧化反应的进一步深入研究和反应机制的全面认识, 底物普适性强、催化活性和选择性更高的小分子仿生催 化剂将会出现.

\section{References}

[1] (a) Katsuki, T. Comprehensive Asymmetric Catalysis I-III: $C-H$ Oxidation, Eds.: Jacobsen, E. N.; Pfaltz, A.; Yamamoto, H., 1999, p. 791.

(b) Costas, M. Coord. Chem. Rev. 2011, 255, 2912.

(c) Newhouse, T.; Baran, P. S. Angew. Chem., Int. Ed. 2011, 50, 3362.

(d) Zhou, M.; Crabtree, R. H. Chem. Soc. Rev. 2011, 40, 1875.

(e) Qian, S.; Ma, Y.; Gao, S.; Luo, J. Chin. J. Org. Chem. 2018, 38, 1930 (in Chinese).

(钱少平, 马尧睿, 高姗姗, 骆钧飞, 有机化学, 2018, 38, 1930.)

(f) Wu, J.; Zhu, J.; Li, H.; Wu, C.; Shen, R.; Yu, L. Chin. J. Org. Chem. 2019, 39, 3328 (in Chinese).

(吴锦雯, 朱佳雯, 李慧, 吴春雷, 沈润溥, 余乐茂, 有机化学, 2019, 39, 3328.)

[2] (a) Li, G.; Zhang, L. Chin. J. Org. Chem. 1985, 5, 27 (in Chinese). (李广年, 张良辅, 有机化学, $1985,5,27$.)

(b) Shen, D.; Miao, C.; Wang, S.; Xia, C.; Sun, W. Org. Lett. 2014, 16, 1108 .

(c) Chen, J.; Lutz, M.; Milan, M.; Costas, M.; Otte, M.; Klein Gebbink, R. J. M. Adv. Synth. Catal. 2017, 359, 2590.

(d) Dantignana, V.; Milan, M.; Cusso, O.; Company, A.; Costas, M.; Bietti, M. ACS Cent. Sci. 2017, 3, 1350.

(e) Milan, M.; Costas, M.; Salamone, M.; Bietti, M. Acc. Chem. Res. 2018, 51, 1984.

(f) Wang, W.; Xu, D.; Sun, Q.; Sun, W. Chem.-Asian J. 2018, 13, 2458.

(g) White, M. C.; Zhao, J. J. Am. Chem. Soc. 2018, 140, 13988.

[3] (a) Guo, M.; Corona, T.; Ray, K.; Nam, W. ACS Cent. Sci. 2019, 5 , 13.

(b) Ray, K.; Pfaff, F. F.; Wang, B.; Nam, W. J. Am. Chem. Soc. 2014, 136, 13942.

(c) Yin, G. Acc. Chem. Res. 2013, 46, 483. 
(d) Que, L. Jr.; Tolman, W. B. Nature 2008, 455, 333.

[4] Luo, Y.-R. Handbook of Bond Dissociation Energies in Organic Compounds, CRC press, Boca Raton, 2003.

[5] Asensio, G.; Castellano, G.; Mello, R.; González Núñez, M. E. J. Org. Chem. 1996, 61, 5564.

[6] Chen, M. S.; White, M. C. Science 2007, 318, 783.

[7] Asensio, G.; González Núñez, M. E.; Bernardini, C. B.; Mello, R.; Adam, W. J. Am. Chem. Soc. 1993, 115, 7250.

[8] Lee, M.; Sanford, M. S. J. Am. Chem. Soc. 2015, 137, 12796.

[9] Luo, F. Chin. J. Org. Chem. 2019, 39, 3084 (in Chinese). (罗飞华，有机化学, 2019, 39, 3084.)

[10] Tenaglia, A.; Terrenova, E.; Waegell, B. J. Org. Chem. 1992, 57, 5523.

[11] Gómez, L.; Garcia-Bosch, I.; Company, A.; Benet-Buchholz, J.; Polo, A.; Sala, X.; Ribas, X.; Costas, M. Angew. Chem., Int. Ed. 2009, 48, 5720 .

[12] Chen, K.; Eschenmoser, A.; Baran, P. S. Angew. Chem., Int. Ed. 2009, 48, 9705 .

[13] Du, X.; Houk, K. N. J. Org. Chem. 1998, 63, 6480

[14] (a) Schlichting, I.; Berendzen, J.; Chu, K.; Stock, A. M.; Maves, S. A.; Benson, D. E.; Sweet, R. M.; Ringe, D.; Petsko, G. A.; Sligar, S. G. Science 2000, 287, 1615 .

(b) Meunier, B.; de Visser, S. P.; Shaik, S. Chem. Rev. 2004, 104, 3947.

(c) Jiang, Y.; Li, S. Chin. J. Org. Chem. 2018, 38, 2307 (in Chinese).

(蒋媛媛, 李盛英, 有机化学, 2018, 38, 2307.)

[15] (a) Ortiz de Montellano, P. R. Chem. Rev. 2010, 110, 932

(b) Abu-Omar, M. M.; Loaiza, A.; Hontzeas, N. Chem. Rev. 2005, 105, 2227.

[16] (a) Groves, J. T.; Viski, P. J. Am. Chem. Soc. 1989, 111, 8537.

(b) Groves, J. T.; Viski, P. J. Org. Chem. 1990, 55, 3628.

[17] Gross, Z.; Ini, S. Org. Lett. 1999, 1, 2077.

[18] Zhang, R.; Yu, W.-Y.; Lai, T.-S.; Che, C.-M. Chem. Commun. 1999, 2441 .

[19] Srour, H.; Le Maux, P.; Simonneaux, G. Inorg. Chem. 2012, 51, 5850 .

[20] Maux, P. L.; Srour, H. F.; Simonneaux, G. Tetrahedron 2012, 68, 5824.

[21] Frost, J. R.; Huber, S. M.; Breitenlechner, S.; Bannwarth, C.; Bach, T. Angew. Chem., Int. Ed. 2015, 54, 691.

[22] (a) Fackler, P.; Berthold, C.; Voss, F.; Bach, T. J. Am. Chem. Soc. 2010, 132, 15911 .

(b) Fackler, P.; Huber, S. M.; Bach, T. J. Am. Chem. Soc. 2012, 134, 12869.

[23] Burg, F.; Gicquel, M.; Breitenlechner, S.; Pöthig, A.; Bach, T. Angew. Chem., Int. Ed. 2018, 57, 2953.

[24] Burg, F.; Breitenlechner, S.; Jandl, C.; Bach, T. Chem. Sci. 2020, 11,
2121.

[25] (a) Katsuki, T. Coord. Chem. Rev. 1995, 140, 189

(b) Bhatia, B.; Punniyamurthy, T.; Iqbal, J. In Asymmetric Oxidation Reactions, Ed.: Katsuki, T., Oxford University Press, New York, 2001, p. 1 $\sim 15$.

(c) Matsumoto, K.; Saito, B.; Katsuki, T. Chem. Commun. 2007, 3619.

(d)Fan, Q.-H.; Ding, K. Top. Organomet. Chem. 2011, 36, 207.

(e) Shaw, S.; White, J. D. Chem. Rev. 2019, 119, 9381.

[26] Hamachi, K.; Irie, I.; Katsuki, T. Tetrahedron Lett. 1996, 37, 4979.

[27] Miyafuji, A.; Katsuki, T. Tetrahedron 1998, 54, 10339.

[28] Punniyamurthy, T.; Miyafuji, A.; Katsuki, T. Tetrahedron Lett. 1998, 39, 8295.

[29] Murahashi, S. I.; Noji, S.; Komiya, N. Adv. Synth. Catal. 2004, 346 , 195.

[30] Murahashi, S.; Noji, S.; Hirabayashi, T.; Komiya, N. Tetrahedron: Asymmetry 2005, 16, 3527.

[31] Milan, M.; Bietti, M.; Costas, M. ACS Cent. Sci. 2017, 3, 196.

[32] (a) Mas-Ballesté, R.; Que, L. Jr. J. Am. Chem. Soc. 2007, 129, 15964

(b) Lyakin, O. Y.; Ottenbacher, R. V.; Bryliakov, K. P.; Talsi, E. P. ACS Catal. 2012, 2, 1196.

[33] (a) Du, J.; Miao, C.; Xia, C.; Lee, Y.-M.; Nam, W.; Sun, W. ACS Catal. 2018, 8,4528 .

(b) Li, X.-X.; Guo, M.; Qiu, B.; Cho, K.-B.; Sun, W.; Nam, W. Inorg. Chem. 2019, 58, 14842 .

[34] Milan, M.; Bietti, M.; Costas, M. Org. Lett. 2018, 20, 2720.

[35] Talsi, E. P.; Samsonenko, D. G.; Ottenbacher, R. V.; Bryliakov, K. P. ChemCatChem 2017, 9, 4580.

[36] Sun, W.; Sun, Q. Acc. Chem. Res. 2019, 52, 2370.

[37] (a) Wang, B.; Miao, C.; Wang, S.; Xia, C.; Sun, W. Chem.-Eur. J. 2012, 18,6750 .

(b) Wang, B.; Wang, S.; Xia, C.; Sun, W. Chem.-Eur. J. 2012, 18, 7332.

(c) Wu, M.; Wang, B.; Wang, S.; Xia, C.; Sun, W. Org. Lett. 2009, 11,3622 .

(d) Miao, C.; Wang, B.; Wang, Y.; Xia, C.; Lee, Y.-M.; Nam, W.; Sun, W. J. Am. Chem. Soc. 2016, 138, 936.

(e) Shen, D.; Qiu, B.; Xu, D.; Miao, C.; Xia, C.; Sun, W. Org. Lett. 2016, 18,372 .

(f) Wang, W.; Sun, Q.; Xia, C.; Sun, W. Chin. J. Catal. 2018, 39, 1463.

[38] Qiu, B.; Xu, D.; Sun, Q.; Miao, C.; Lee, Y.-M.; Li, X.-X.; Nam, W.; Sun, W. ACS Catal. 2018, 8, 2479.

[39] Qiu, B.; Xu, D.; Sun, Q.; Lin, J.; Sun, W. Org. Lett. 2019, 21, 618.

[40] Cianfanelli, M.; Olivo, G.; Milan, M.; Klein Gebbink, R. J. M.; Ribas, X.; Bietti, M.; Costas, M. J. Am. Chem. Soc. 2020, 142, 1584.

(Cheng, F.) 